\title{
Resultados preliminares do uso de anti-hiperglicemiantes orais no diabete melito gestacional
}

\author{
Preliminary results of the use of oral hypoglycemic drugs on gestational diabetes mellitus \\ Jean Carl Silva ${ }^{1}$, Wladimir Taborda ${ }^{2}$,Felipe Becker ${ }^{3}$, Gabriela Aquim $^{3}$, Juliana Viese ${ }^{3}$, Anna Maria Bertini ${ }^{4}$
}

\section{RESUM0}

Objetivo: comparar a eficácia da glibenclamida e da acarbose com insulina no tratamento do diabete melito gestacional (DMG) em relação ao controle glicêmico materno, peso do recém-nascido (RN) e hipoglicemia neonatal. Métodos: trata-se de ensaio clínico randomizado, prospectivo e aberto. Foram incluídas 57 pacientes com diagnóstico de DMG, que necessitaram de terapêutica complementar à dietoterapia e à atividade física. As gestantes foram aleatoriamente alocadas em um de três grupos com terapêuticas diferentes: um grupo controle conduzido com insulinoterapia, outro com glibenclamida e outro com acarbose. O período do estudo foi de sete meses ( $1^{\circ}$ de outubro de 2003 a $1^{\circ}$ de maio de 2004). Os desfechos primários avaliados foram o nível glicêmico materno após o inicio do tratamento, a necessidade de troca de terapêutica para controle glicêmico, peso do RN e presença de hipoglicemia neonatal. A análise estatística foi realizada pelo teste estatístico ANOVA, com nível de significância de 5\%. Resultados: as características maternas foram semelhantes nos três grupos estudados. O controle glicêmico não foi obtido em três pacientes que utilizaram glibenclamida (15\%) e em sete das usuárias de acarbose $(38,8 \%)$. Não houve diferença quanto à glicemia em jejum e pós-prandial e no peso médio do RN entre os três grupos. A incidência de fetos grandes para a idade gestacional foi de 5,2, 31,5 e 11,1\% nos grupos tratados com insulina, glibenclamida e acarbose, respectivamente. A hipoglicemia neonatal ocorreu em seis RN, sendo quatro deles do grupo glibenclamida (21,0\%). Conclusões: a glibenclamida foi mais eficiente para o controle glicêmico que a acarbose, mas ambos foram menos eficientes que a insulina. Os RN de pacientes alocadas no grupo glibenclamida apresentaram maior incidência de macrossomia e de hipoglicemia neonatal quando comparados com os RN cujas mães receberam outros tratamentos.

PALAVRAS-CHAVE: Diabetes gestacional/quimioterapia; Glicemia/efeito de drogas; Macrossomia fetal

\section{ABSTRACT}

Purpose: to compare the effectiveness of glibenclamide and acarbose with that of insulin for the treatment of gestational diabetes mellitus (GDM), in regard to maternal glucose levels, newborn (NB) weight and neonatal hypoglycemia. Methods: an open, randomized prospective study was carried out. Fifty-seven patients diagnosed with GDM were included. These patients required dietary control and additional therapy. Pregnant women were randomly alloted to one of three groups with different therapies: a control group making use of insulin therapy, a study group making use of glibenclamide and a study group making use of acarbose. The study took seven months (from October 1st 2003 to May 1st 2004). Assessed outcomes were maternal glucose levels in the prenatal period, the need for replacing therapy to achieve glucose level control, NB weight and neonatal hypoglycemia. Statistical analysis was determined by ANOVA with the level of significance set at $5 \%$. Results: maternal characteristics were similar in all the three groups. Glucose level control was not obtained in three of the patients who used glibenclamide (15\%) and in seven (38.8\%) of the patients who used acarbose. Regarding fasting and postprandial glucose level rates and average NB weight no difference between the three groups was observed. No statistical difference was found for fasting or postprandial glucose levels and average NB weight in any of the three groups. The rate of large for gestational age fetuses was 5.2, 31.5 and $11.1 \%$ for the groups treated with insulin, glibenclamide and acarbose, respectively. Neonatal hypoglycemia was observed in six NB. Four of these were from the glibenclamide group (21.0\%). Conclusions: glibenclamide was more effective for glucose level control than acarbose but neither were more efficient than insulin. NB children whose mothers had been alloted to the glibenclamide group showed a higher rate of macrosomia and neonatal hypoglycemia when compared to those newborns whose mothers were subjected to other therapies.

KEYWORDS: Diabetes gestacional/drug therapy; Blood glucose/drug effects; Fetal macrosomia

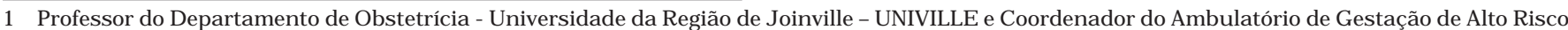
da Maternidade Darcy Vargas - Joinville (SC), Brasil.

2 Médico Coordenador do Serviço de Perinatologia do Hospital Israelita Albert Eisntein - São Paulo (SP), Brasil.

3 Academico do Curso de Medicina da Universidade da Região de Joinvile - UNIVILLE - Joinvile (SC), Brasil.

4 Professor Associado do Departamento de Obstetrícia - Universidade Federal de São Paulo - UNIFESP - São Paulo (SP), Brasil.

Correspondência: Jean Carl Silva - TEGO 0143/1992

Rua Oscar Schneider 205, casa 10 - 89203-050 - Joinville - SC - Fone (47) 422-1356 Fax (47) 422-6912 - e-mail: Jeancs01@terra.com.br 


\section{Introdução}

O diabete melito gestacional (DMG) é definido como qualquer grau de intolerância à glicose, com aparecimento ou primeiramente descoberta durante a gestação. A definição se aplica tanto para a gestante que usa insulina, como apenas com o tratamento por meio de dieta, e com persistência ou não da doença após a gestação. Não exclui a possibilidade de intolerância à glicose não reconhecida previamente ou o início concomitante com a gestação ${ }^{1}$.

A incidência do DMG varia amplamente, oscilando entre 1 e 14\%, dependendo da população estudada e do método utilizado para rastreamento e diagnóstico. A incidência do DMG em mulheres com mais de 20 anos atendidas no Sistema Único de Saúde (SUS) é de 7,6\% $\%^{2}$.

A mortalidade perinatal é quatro vezes maior entre os filhos de gestantes com DMG, e a morbidade perinatal também está aumentada, com altos índices de macrossomia, tocotraumatismo e complicações metabólicas ${ }^{3}$.

O tratamento do diabete durante a gestação visa um bom controle glicêmico. Quando ocorre falha na obtenção de bom controle glicêmico com dieta, associada ou não à exercícios físicos, está indicada a insulinoterapia ${ }^{4-6}$. Em torno de 15 a $60 \%$ das pacientes com diagnóstico de DMG necessitam de tratamento com insulina ${ }^{7-9}$.

A insulina é terapia efetiva para controlar a glicemia materna, porém cara e com manejo inconveniente. Muitos autores não recomendam o uso das sulfoniluréias durante a gestação, devido ao aumento na incidência de anomalias fetais e de hipoglicemia neonatal. Esta recomendação é baseada principalmente em estudos feitos antes que drogas novas como a glibenclamida estivessem disponíveis ${ }^{10}$.

A contra-indicação para o uso de antihiperglicemiantes orais na gestação foi baseada em relatos de casos e estudos com pequenas amostras, que sugeriam efeitos adversos sobre o desenvolvimento do feto ${ }^{11}$. Um estudo randomizado demonstrou que não existe associação entre antihiperglicemiantes orais e malformações fetais, concluindo que as sulfoniluréias são seguras para uso na gestação ${ }^{12}$.

A glibenclamida é uma sulfoniluréia de segunda geração e foi aprovada pelo FDA para uso humano em 1984. Sua ação anti-hiperglicemiante se deve a aumento da liberação da insulina pelo pâncreas, porém efeitos extrapancreáticos devem estar envolvidos ${ }^{13}$.

A glibenclamida não cruza a barreira placentária, não altera os níveis de insulina fetal, não está associada ao aumento da mortalidade perinatal e apresenta resultados perinatais iguais aos obtidos com insulina. Os resultados foram obtidos por um ensaio clínico randomizado com mais de quatrocentas gestantes com DMG, sendo o trabalho mais importante até o momento ${ }^{10}$.

A transferência da glibenclamida através da barreira placentária é insignificante ${ }^{14}$. As causas desta baixa transferência são a ligação com as lipoproteínas (99,8\%), permanecendo apenas 0,2\% da droga livre para cruzar a barreira placentária. Este fato, associado à meia-vida curta (4 horas), explica a não detecção no sangue fetal logo após o parto $^{10,15}$.

A acarbose é um pseudotetrassacarídeo solúvel em água, que atrasa a digestão dos carboidratos ingeridos e sua distribuição, resultando em elevação menos acentuada da glicemia pós-prandial, reduzindo, assim, a glicemia plasmática ${ }^{16}$. Exerce controle sobre a glicemia materna, é bem tolerada e não causa alterações fetais $^{17,18}$.

A glibenclamida, a acarbose e a insulina são classificadas como classe B para uso durante a gestação, isto é, sem efeitos deletérios para o feto. Isto foi demonstrado em pesquisas animais, não havendo estudos controlados que mostrem algum efeito nocivo em humanos, sem evidências de risco após o primeiro trimestre ${ }^{13}$.

Além do custo elevado da insulinoterapia, temos a alta complexidade em relação ao seu uso e a rejeição das pacientes ao tratamento. Uma opção mais barata, simples e de fácil aceitação, como o uso de terapêutica oral, torna-se de grande interesse, não só para a saúde pública, como para toda a comunidade médica. Estudar alternativas como o uso dos anti-hiperglicemiantes orais para o tratamento do DMG é o objetivo deste trabalho.

\section{Pacientes e Métodos}

O desenho de pesquisa foi do tipo ensaio clínico prospectivo, aleatório e aberto. Foram incluídas 57 pacientes com diagnóstico de DMG que necessitaram de terapêutica complementar à dietoterapia e à atividade física. As gestantes foram aleatoriamente alocadas em um de três grupos com terapêuticas diferentes: um grupo controle conduzido com insulinoterapia $(n=19)$, outro com glibenclamida $(n=19)$ e outro com acarbose $(n=18)$. O período de inclusão no estudo foi de sete meses, entre $1^{\circ}$ de outubro de 2003 e $1^{\circ}$ de maio de 2004 .

O local do estudo foi a Maternidade Darcy Vargas (MDV), localizada na cidade de Joinville, 
na qual são atendidas quase que exclusivamente pacientes do SUS. A mesma possui um serviço multidisciplinar de atendimento a gestantes portadoras de diabete, contando com nutricionista, fisioterapeuta, psicóloga, enfermeira e obstetra. Foram incluídas pacientes com diagnóstico de DMG, que necessitaram de terapêutica complementar à dietoterapia e atividade física, com idade gestacional no momento do diagnóstico entre 11 e 33 semanas e gestações únicas. O diagnóstico de DMG foi estabelecido com o uso de glicemia de jejum para rastreamento e teste de tolerância oral à glicose com $75 \mathrm{~g}$ (TTOG $75 \mathrm{~g}$ ) conforme orientação do Ministério da Saúde ${ }^{19}$.

O acompanhamento das pacientes foi efetuado conforme rotina do Serviço ${ }^{20}$, incluindo dietoterapia e atividade física por período de três a sete dias, sendo feito, a seguir, um perfil glicêmico para avaliar a necessidade de tratamento complementar. Os valores limites do perfil glicêmico foram: glicemia em jejum (90 mg/dL), duas horas após o café (100 mg/dL), duas horas após almoço (100 mg/dL) e duas horas após o jantar (100 mg/ $\mathrm{dL})^{21}$. Se o perfil glicêmico estivesse alterado, era sugerida a participação no estudo. As glicemias foram realizadas com o uso do método enzimático automatizado, no aparelho Airomi 200, da Winner ${ }^{\oplus}$.

Foi realizado sorteio simples, mediante envelope pardo contendo em seu interior o grupo ao qual a paciente seria incluída, e com regime aberto após o sorteio, em função das diferentes vias e modos de administração das terapêuticas. O tamanho amostral não foi calculado, e os resultados são preliminares.

Todas as pacientes foram acompanhadas ambulatorialmente com perfil glicêmico de sete em sete dias até o controle glicêmico, após foi realizada a cada consulta, uma glicemia em jejum e pós-prandial de duas horas. Se um ponto estivesse alterado, colhiam-se novas amostras de sangue às 17 e 19 horas para completar o perfil glicêmico e reajustar a dose do tratamento selecionado. Todos os valores coletados após 7 dias do início da terapêutica foram considerados no desfecho primário do controle glicêmico. Se atingida a dose máxima do anti-hiperglicemiante oral sem a obtenção do controle glicêmico, a terapia era substituída por insulinoterapia ${ }^{10}$.

O grupo controle utilizou insulina, sendo a gestante rotineiramente internada para aprendizagem por um período de 24 horas. A dose total administrada foi baseada no peso atual e na idade gestacional materna $(0,7 \mathrm{UI} / \mathrm{kg}$ no primeiro trimestre, $0,8 \mathrm{UI} / \mathrm{kg}$ no segundo trimestre e 0,9 UI/ $\mathrm{kg}$ no terceiro trimestre). Utilizamos insulina humana de ação rápida (regular) antes das principais refeições e de ação lenta (NPH) ao deitar, divididas em doses iguais inicialmente.

Outro grupo utilizou glibenclamida, ambulatorialmente. A dose inicial foi de $5 \mathrm{mg}$ pela manhã, com dose máxima de $20 \mathrm{mg}$ ao dia. A mesma foi fornecida gratuitamente pelo SUS. O terceiro grupo utilizou acarbose $50 \mathrm{mg}$ antes das principais refeições, com dose máxima total de 300 $\mathrm{mg}$ ao dia. Esta foi fornecida gratuitamente pelo laboratório produtor da medicação, sem retorno financeiro.

Foi sugerida a interrupção da gestação, com idade gestacional máxima de 39 semanas ${ }^{4}$. Foi realizado controle glicêmico durante o trabalho de parto com insulina regular, mantendo-se a glicemia em valores inferiores a $120 \mathrm{mg} / \mathrm{dL}$. A interrupção da gestação foi realizada pelo médico plantonista da instituição e a indicação da via de parto foi obstétrica.

Os dados maternos avaliados incluíram escolaridade, idade, paridade, idade gestacional do diagnóstico de DMG (com base na primeira ultrasonografia realizada pela paciente), tratamento (anti-hiperglicemiante oral ou insulina), doses utilizadas no tratamento, idade gestacional de término da gravidez, hipoglicemias graves que necessitaram internação, via de parto e outras intercorrências não especificadas.

Os RNs foram avaliados durante o período de internação. Os dados avaliados incluíram peso ao nascer, macrossomia (peso superior a $4000 \mathrm{~g}$ ) e RN classificados como grande para a idade gestacional - GIG (peso superior ao percentil 90 na curva de crescimento de Lubchenko) ${ }^{22}$.

A glicemia capilar sistêmica dos RN foi realizada na primeira, terceira e sexta hora pós-nascimento, exceto se havia outra indicação clínica, e a hipoglicemia neonatal foi considerada para valores inferiores a $40 \mathrm{mg} / \mathrm{dL}$. Outras intercorrências neonatais avaliadas incluíram o número de dias de internação, necessidade de UTI neonatal, óbito e condições de alta.

Os dados foram armazenados no programa Access da Microsoft e a análise estatística foi realizada pelo programa Excell da Microsoft, pelo teste estatístico ANOVA, com nível de significância de 5\%.

Os desfechos primários avaliados foram nível glicêmico materno avaliado sete dias após o inicio do tratamento complementar até a consulta anterior ao parto, necessidade de troca de terapêutica para controle glicêmico, peso do RN e ocorrência de hipoglicemia neonatal. O número citado em cada grupo é o inicial e não foram excluídos da análise os casos com falhas terapêuticas em cada grupo. 
A pesquisa foi realizada após autorização da Comissão de Ética da Maternidade Darcy Vargas, sendo oferecido um consentimento livre e esclarecido, conforme a resolução 196/96 do Conselho Nacional de Saúde ${ }^{23}$.

\section{Resultados}

No período de inclusão 124 pacientes com diabete associado à gestação iniciaram seu prénatal e tiveram seus filhos na Maternidade Darcy Vargas. Desse total, 57 foram incluídas no presente estudo, sendo que uma paciente foi excluída posteriormente em função de quadro asmático grave, que exigiu corticoterapia para controle clínico. Não houve diferença entre os três grupos quanto à idade, número de gestações, índice de massa corpórea, data de inclusão no estudo e idade gestacional no momento do parto (Tabela 1).

Tabela 1 - Perfil epidemiológico das gestantes com DMG alocadas para diferentes formas de tratamento (médias \pm desvio-padrão).

\begin{tabular}{lrrrr}
\hline & $\begin{array}{c}\text { Insulina } \\
(\mathbf{n}=19)\end{array}$ & $\begin{array}{c}\text { Glibenclamida } \\
(\mathbf{n}=19)\end{array}$ & $\begin{array}{c}\text { Acarbose } \\
(\mathbf{n}=\mathbf{1 8})\end{array}$ & Valor - $\mathbf{p}$ \\
\hline Idade & $29,7 \pm 5,5$ & $32,1 \pm 4,0$ & $31,5 \pm 6,0$ & 0,36 \\
Gesta & $2,9 \pm 1,8$ & $3,4 \pm 2,7$ & $2,8 \pm 1,7$ & 0,66 \\
IMC & $27,0 \pm 7,8$ & $27,6 \pm 5,9$ & $25,8 \pm 4,3$ & 0,67 \\
IG início & $26,2 \pm 5,6$ & $25,3 \pm 9,5$ & $27,1 \pm 7,4$ & 0,77 \\
IG parto & $38,1 \pm 1,2$ & $37,9 \pm 1,4$ & $38,1 \pm 1,2$ & 0,56 \\
\hline
\end{tabular}

Gesta: número de gestações.

IMC: índice de massa corporal.

IG início: idade gestacional de início do tratamento.

IG parto: idade gestacional no momento do parto.

Em relação ao controle glicêmico, verificamos que a maioria das pacientes do grupo da glibenclamida (10 casos que representaram $52,6 \%$ dessa amostra) teve as suas glicemias normalizadas com a dose inicial de $5 \mathrm{mg}$ ao dia. No entanto, somente quatro pacientes que utilizaram acarbose (22\% desse grupo) obtiveram controle glicêmico adequado com a dose inicial da droga (150 mg). Nenhuma paciente necessitou internação hospitalar para controle de hipoglicemia.

O controle glicêmico não foi obtido em três pacientes do grupo glibenclamida $(15,7 \%)$ e em sete das usuárias de acarbose (38,8\%). Não houve diferença entre as glicemias em jejum e pósprandiais nos três grupos. Os resultados maternos e neonatais estão agrupados na Tabela 2.
Tabela 2 - Médias dos resultados \pm respectivos desvios-padrão das glicemias de jejum e pós-prandiais, obtidas durante todo tratamento, das gestantes, e média e desviospadrão dos pesos de seus neonatos logo após o nascimento.

\begin{tabular}{cccc}
\hline $\begin{array}{c}\text { Insulina } \\
(n=19)\end{array}$ & Glibenclamida & Acarbose & Valor $-p$ \\
$(n=19)$ & $(n=18)$ & \\
\hline
\end{tabular}

GJmédia $\quad 84,8 \pm 8,5 \mathrm{mg} / \mathrm{dL} \quad 92,6 \pm 20,7 \mathrm{mg} / \mathrm{dL} \quad 87,9 \pm 8,5 \mathrm{mg} / \mathrm{dL} \quad 0,22$

GP média 100,6 $\pm 17,4 \mathrm{mg} / \mathrm{dL} \quad 101,8 \pm 20,0 \mathrm{mg} / \mathrm{dL} \quad$ 107,1 $\pm 19,0 \mathrm{mg} / \mathrm{dL} \quad 0,55$

Pesofetal $3.112,3 \pm 440,7 \mathrm{~g} \quad 3.378,5 \pm 584,8 \mathrm{~g} \quad 3.249,4 \pm 441,1 \mathrm{~g} \quad 0,24$

GJ: média das glicemias de jejum durante o pré-natal.

GP: média das glicemias pós-prandiais durante o pré-natal.

A incidência de fetos GIG foi de 5,2, 31,5 e $11,1 \%$ nos grupos tratados com insulina, glibenclamida e acarbose, respectivamente. A macrossomia foi encontrada apenas no grupo da glibenclamida, em quatro RN (21\%). A hipoglicemia neonatal ocorreu em seis RN: nos grupos da acarbose e da insulina tivemos apenas um caso em cada, e no grupo da glibenclamida tivemos quatro casos (21\%). Apenas um RN com 36 semanas, filho de gestante que utilizou glibenclamida, necessitou de cuidados especiais por período de dois dias, em uma unidade intermediária, por hipoglicemia prolongada.

Não ocorreram óbitos perinatais e todos os RNs tiveram Apgar no $5^{\circ}$ minuto maior ou igual a 7. Dois RN foram classificados como pequenos para idade gestacional. A incidência de cesariana foi igualmente elevada nos três grupos $(47 \%$ nos grupos da insulina e glibenclamida e 55\% no grupo acarbose). Nenhum caso de tocotraumatismo foi observado.

\section{Discussão}

A eficácia dos anti-hiperglicemiantes orais pode ser avaliada pelas glicemias médias, em jejum e pós-prandiais, após a instituição da terapêutica, observando-se resultados semelhantes aos obtidos com o uso da insulina, na maioria das pacientes ${ }^{24}$. O controle glicêmico é de especial importância para a redução das complicações perinatais, sendo o desfecho mais importante. Em nosso material, a necessidade de mudança da terapêutica para insulinoterapia ocorreu em sete pacientes que utilizaram acarbose $(38,8 \%)$ e em três no grupo da glibenclamida (15,7\%), ratificando que a acarbose é droga menos efetiva que a glibenclamida para o controle glicêmico. Em outros estudos a falha de controle glicêmico com glibenclamida variou entre 4 e $19 \%^{10,25-27}$. De forma similar a outro estudo ${ }^{10}$, não verificamos nenhum caso de hipoglicemia materna que neces- 
sitasse internação hospitalar em nenhum dos grupos.

No grupo da glibenclamida houve maior incidência de macrossomia (21\%) e de GIG (31\%) em relação aos grupos acarbose e insulina. De forma conflitante, alguns autores encontraram maior incidência de macrossomia no grupo da insulina e menor em seu grupo tratado com glibenclami$\mathrm{da}^{28}$. Outros autores não encontraram diferença entre o grupo tratado com glibenclamida e insulina ${ }^{10}$. Em um estudo na África do Sul, foi encontrada incidência de GIG semelhante à nossa $(30 \%)^{29}$. Comparando insulina e acarbose, alguns autores encontraram RN menores no grupo da acarbose e macrossomia presente em 5\% das tratadas com acarbose, ao passo que $21 \%$ das gestantes que utilizaram insulina tiveram RN macrossômicos ${ }^{18}$. A incidência de macrossomia em nosso estudo nas gestantes usuárias de glibenclamida foi semelhante à encontrada por autores nacionais, que trataram as gestantes com DMG de forma tradicional, isto é, com dieta, atividade física e insulina quando necessário ${ }^{30}$.

Não verificamos diferença no peso médio dos $\mathrm{RN}$ nos três grupos. Outros autores também não encontraram em seus estudos diferença no peso fetal entre o grupo que utilizou anti-hiperglicemiante oral e insulina ${ }^{10,24,28}$. Tivemos dois casos de RN PIG no grupo da insulina (10,5\%), semelhante à literatura ${ }^{28}$.

A hipoglicemia neonatal também ocorreu em maior número no grupo da glibenclamida (21\%). Outros autores também tiveram maior incidência dessa morbidade no grupo da glibenclamida $(26,7 \%)$ quando comparado ao grupo da insulina (7\%), todos os casos com fácil tratamento ${ }^{28}$. No nosso grupo tratado com glibenclamida, apenas um RN necessitou de cuidados especiais em uma unidade intermediária, por período de 2 dias, diferente de outros autores que não encontraram nenhum caso de hipoglicemia grave ${ }^{24}$ e não observaram diferença na incidência de hipoglicemia neonatal ${ }^{10}$.

A DMG aumenta a incidência de óbito perinatal quando comparada à população geral. Alguns autores, analisando tratamento convencional, encontraram $3,7 \%$ de óbitos perinatais nas gestantes com $\mathrm{DMG}^{29}$. Não tivemos nenhum caso de óbito perinatal.

Com estes resultados preliminares, consideramos que a glibenclamida e a acarbose podem representar alternativas terapêuticas promissoras para o tratamento do DMG. A glibenclamida controlou a glicemia na maioria das pacientes e foi mais eficiente que a acarbose, mas ambas foram menos eficientes que a insulina. A gliben- clamida apresentou maior incidência de macrossomia e hipoglicemia neonatal quando comparada com os outros tratamentos, resultado similar à literatura internacional, porém sem repercussões clínicas neonatais relevantes.

Finalmente, os resultados clínicos preliminares obtidos com a utilização de anti-hiperglicemiantes orais em gestantes diabéticas devem ser avaliados em estudos com maior tamanho amostral, de modo a corroborar os achados da literatura atual, que ainda está limitada a ensaios com casuísticas reduzidas.

\section{Conflito de interesses}

Nenhum dos autores possui qualquer vínculo ou relação com as empresas produtoras dos equipamentos ou drogas citadas, assim como concorrentes, não recebendo qualquer tipo de auxílio. Não há conflito de interesses.

\section{Referências}

1. American Diabetes Association. Diagnosis and classification of diabetes mellitus. Diabetes Care. 2005;28 Suppl 1:S37-42.

2. Ministério da Saúde. Secretaria de Políticas de Saúde. Gestação de alto risco. 3a ed. Brasília (DF): SPS; 2000.

3. Jacobson JD, Cousins L. A population-based study of maternal and perinatal outcome in patients with gestational diabetes. Am J Obstet Gynecol. 1989;161(4):981-6.

4. Landon MB, Gabbe SG. Diabete mélito. In: Barron WM, Lindhemeir MD, editores. Complicações médicas na gravidez. 2a ed. Porto Alegre: Artes Médicas; 1996. p. 55-77.

5. Rotondo L, Coustan DR. Diabete mélito na gestação. In: Knuppel RA, Drukker JE, editores. Alto risco em obstetrícia: um enfoque multidisciplinar. $2^{a}$ ed. Porto Alegre: Artes Médicas; 1996. p. 424-38.

6. Bertini AM. Diabetes mellitus e gravidez. In: Federação Brasileira de Ginecologia e Obstetrícia. Tratado de obstetrícia. São Paulo: Atheneu; 2000. p. 566-637.

7. Homko CJ, Reece EA. Ambulatory care of the pregnant woman with diabetes. Clin Obstet Gynecol. 1998;41(3):584-96.

8. Aberg A, Rydhstroem H, Frid A. Impaired glucose tolerance associated with adverse pregnancy 
outcome: a population-based study in southern Sweden. Am J Obstet Gynecol. 2001;184(2):77-83.

9. Langer O. Oral hypoglycemic agents and pregnant diabetic: "from bench to bedside". Semin Perinatol. 2002;26(3):215-24.

10. Langer O, Conway DL, Berkus MD, Xenakis EM, Gonzales O. A comparison of glyburide and insulin in women with gestational diabetes mellitus. N Engl J Med. 2000;343(16):1134-8.

11. Piacquadio K, Hollingsworth DR, Murphy H. Effects of in-utero exposure to oral hypoglycaemic drugs. Lancet. 1991;338(8771):866-9.

12. Notelovitz M. Sulphonylurea therapy in the treatment of the pregnant diabetic. S Afr Med J. 1971;45(9):226-9.

13. Larimore WL, Petrie KA. Drug use during pregnancy and lactation. Update in maternity care. Prim Care. 2000;27(1):35-53.

14. Elliot BD, Langer O, Schenker S, Johnson RF. Insignificant transfer of glyburide occurs across the human placenta. Am J Obst Gynecol. 1991;165(4 Pt 1):807-12.

15. Koren G. Glyburide and fetal safety; transplacental pharmacokinetic considerations. Reprod Toxicol. 2001;15(3):227-9.

16. Ford MD, Delaney KA, Ling LJ, Erickson T. Clinical toxicology. Philadelphia: W.B. Saunders; 2001.

17.Zárate A, Ochoa R, Herández M, Basurto L. Eficacia de la acarbose para controlar el deterioro de la tolerancia a la glucosa durante la gestación. Ginecol Obstet Mex. 2000;68:42-4.

18. Platt J, O’Brien W. Acarbose therapy for gestational diabetes: a retrospective cohort study. Am J Obstet Gynecol. 2003;189(6 Suppl):S107.

19. Ministério da Saúde. Diabetes gestacional. Manual de hipertensão arterial e diabetes mellitus. Brasília (DF): Editora MS; 2002.

20. Silva JC. Gestante diabética. Rio de Janeiro: EPUB; 2003.
21. Coustan DR, Berkowitz RL, Hobbins JC. Tight metabolic control of overt diabetes in pregnancy. Am J Med. 1980;68(6):845-52.

22. Oppermann MLR, Reichelt AJ. Diabete mélito e gestação. In: Freitas F, Martins-Costa SH, Ramos JGL, Magalhães JA, editores. Rotinas em obstetrícia. 4a ed. Porto Alegre: Artmed; 2001. p. 407-28.

23. Conselho Nacional de Saúde (CNS). Diretrizes e normas regulamentadoras de pesquisas envolvendo seres humanos. Resolução 196/96. Brasília (DF): CNS; 1996.

24. Hellmuth E, Damm P, Molsted-Pedersen L. Oral hypoglycaemic agents in 118 diabetic pregnancies. Diabet Med. 2000;17(7):507-11.

25. Kremer CJ, Duff P. Glyburide for the treatment of gestational diabetes. Am J Obstet Gynecol. 2004;190(5):1438-9.

26. Chmait R, Dinise T, Moore T. Prospective observational study to establish predictors of glyburide success in women with gestational diabetes mellitus. J Perinatol. 2004;24(10):617-22.

27. Conway DL, Gonzales O, Skiver D. Use of glyburide for the treatment of gestational diabetes: the San Antonio experience. J Matern Fetal Neonatal Med. 2004;15(1):51-5.

28. Coetzee EJ, Jackson WP. Oral hypoglycaemics in the first trimester and fetal outcome. S Afr Med J. 1984;65(16):635-7.

29. Daponte A, Guidozzi F, Moisuc D, Marineanu A. Management of diabetic pregnant patients in a tertiary center in the developing world. Int $\mathrm{J}$ Gynaecol Obstet. 1999;64(2):141-6.

30. Montenegro Junior RM, Paccola GMFG, Faria CM, Sales APM, Montenegro APDR, Jorge SM, et al. Evolução materno-fetal de gestantes diabéticas seguidas no HC-FMRP-USP no período de 1992-1999. Arq Bras Endocrinol Metab. 2001;45(5):467-74. 